\begin{tabular}{ccc}
\hline & International Journal of Engineering \& Technology, $9(1)(2020) 26-34$ \\
SPC & Website: www.sciencepubco.com/index.php/IJET \\
Research paper & Jechnology \\
\hline
\end{tabular}

\title{
Designed and Implementing of Quadcopter Control Unit Capable of Tracking and Detecting Airplanes Types
}

\author{
Mehdi Aslinezhad $^{1} *$, Massoud Hosseinzadeh ${ }^{2}$, Farsad Heidari $^{3}$ \\ ${ }^{1}$ Department of Electrical Engineering, Shahid Sattari Aeronautical University of Science and Technology, Tehran 1384663113, Iran \\ ${ }^{2}$ Babol Noshirvani University of Technology, Babol, Iran \\ ${ }^{3}$ Department of Electrical Engineering, Shahid Sattari Aeronautical University of Science and Technology, Tehran, Iran \\ *Corresponding author E-mail: M.aslinezhad@ssau.ac.ir
}

\begin{abstract}
The goal of this study is to design and implement a new idea for air-launched quadcopter control unit using the digital video processing. In this study, it's endeavored to transmit the captured videos by a camera embedded on the quadcopter via video transceiver and after processing, the ground station relays appropriate command to the quadcopter to tracking target. Moreover, it's desired to detect a target in the sky and both stay in one direction until detect the target when intercepting toward the target and the quadcopter intercepts the target in every direction. In order to identify the type of aerial targets, the dimensions are studied at different intervals, which makes military and non - military goals detected at different intervals.
\end{abstract}

Keywords: Quadcopter; Digital Video Processing; Video and Data Transceiver.

\section{Introduction}

Air defense is responsible for protecting wealth, resources and assets of marine and land. There are several types of air threats including: surface-to-air missiles, surface-to-surface missiles and drones and aircrafts. Several missile and air defense systems are used for dealing with these threats.

The aim of present study, is tracking and chasing the air target by means of heat detector sensor in a cloudy and clear sky [1].

Targets studied in the present study are tracking an air target using camera and using MATLAB software, which after receiving images, running two engines of $\mathrm{x}$-axis airfoils and results in guiding missile toward the target [2].

In the present study, a missile has been tracked in the air using frame and its speed and a rectangular box is embedded in the exhausted gases section, so that the missile speed is estimated by using this box [3].

In this section, it is tried to tracking and chasing a target using camera and joystick. First, after turning the camera using joystick for searching a target, the surrounding is searched and then by appearing the target it locked on it and then missile is involved with target [4]. In the present study, a new image processing-based system was presented in order to automatic guidance of quadcopter. This system is composed of a camera embedded on the quadcopter, video and data transmitter with several kilometers ranges. The video from quadcopter is transmitted to portable computer system on the earth by transmitter and after processing the received video using software, the commands are again sent to quadcopter using long-range data transmitter for moving the quadcopter brushless motors and tracking the target. SMD, light weight and cheap components were used in the presented design. The aim of presented designs is the capability of detecting the type of air targets and automatic tracking target with help of video processing. Figure 1 shows the overall view of recommended system. Images are recorded by camera embedded on the quadcopter and are transmitted to computer system on the earth by long-range transmitter. After processing the image, the required commands are transmitted back to quadcopter using long-range data transmitter in order to guiding quadcopter. With this method, can be controlled and guiding operation can be done with high precision. Quadcopter has a camera with video signal output and PAL system. 

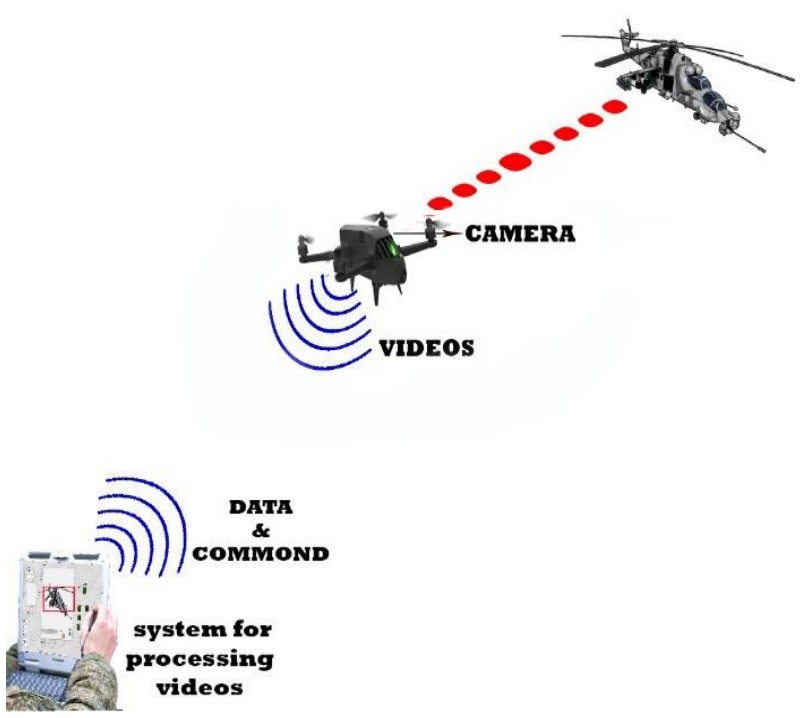

Fig. 1: Overall View of Recommended System.

In the present study, a system was designed, implemented and investigated based on the recommended items. The recorded images of target in sight of quadcopter, by camera embedded on the quadcopter, are transmitted online to a computer system in the base station. These images are processed using LABVIEW software. This software can transmit codes, which include "X" and " $Y$ " axis of target on the system screen, using APC220 wireless transmitter and receiver thorough USB port to Arduino MEGA. After receiving transmitted codes which include parameters related to " $\mathrm{X}$ " and "Y" axis, brushless motors turned on in order to tracking target by quadcopter; it means that, by each movement of target, by help of processing of transmitted images and the movement of brushless motors based on the received codes, quadcopter moves toward the target and locked on the target. Figure 2 shows the system operation during these steps from receiving transmitted images to retransmitting command to quadcopter. Figure 3 shows the overall architecture of the system.

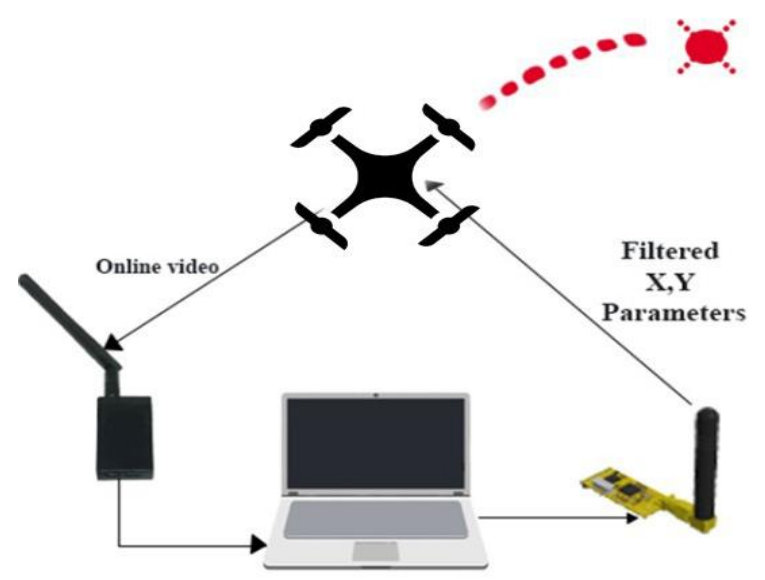

Central Data Processing

Fig. 2: Overall Operation of System.

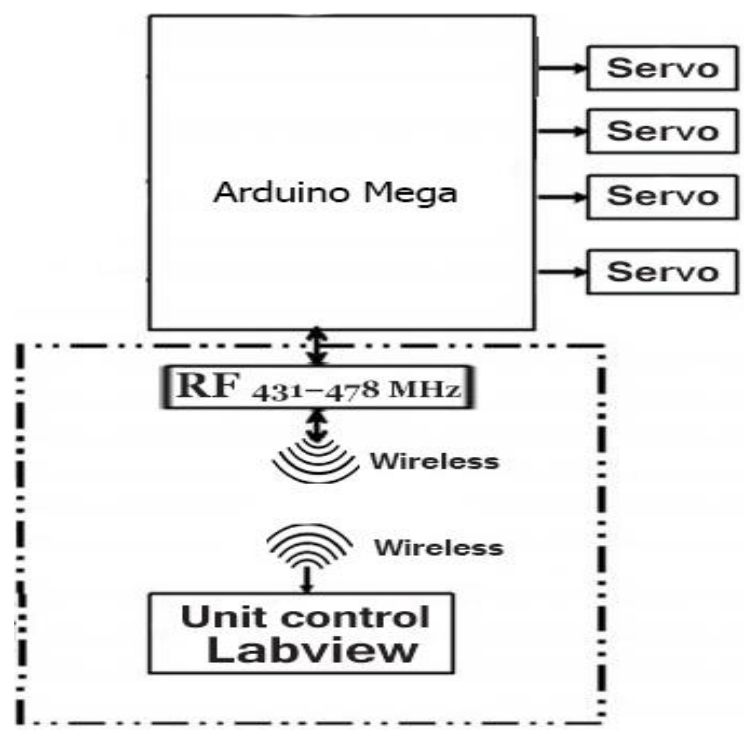

Fig. 3: Architecture of System Control Part (Quadcopter Control Part). 
Figure 4 shows the architecture of system images wireless transmitter-receiver part.

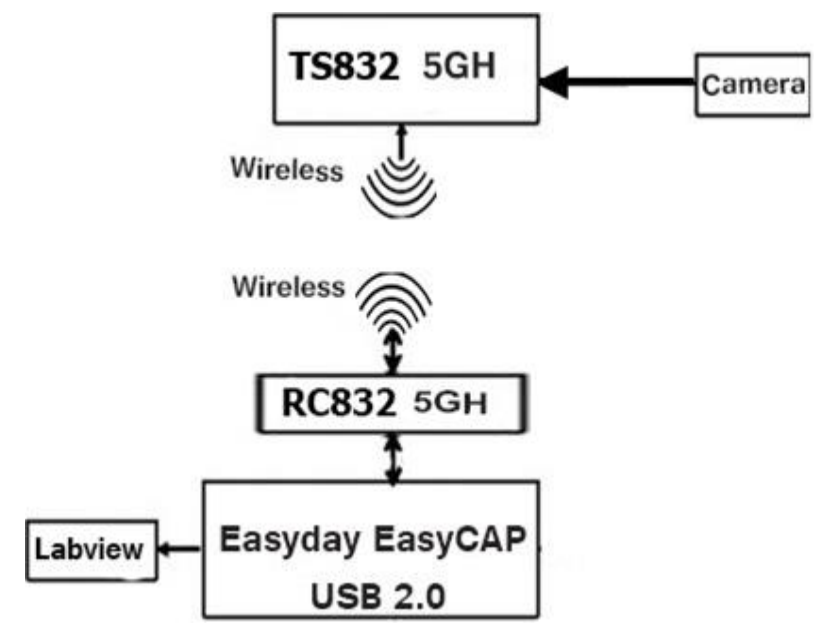

Fig. 4: Architecture of System Images Wireless Transmitter-Receiver Part.

Commands of each of "X" and "Y" axis are transmitted to quadcopter by APC220 data transmitter-receiver. Working frequency of each of these transmitter and receiver is different; for example, working frequencies of "X" and "Y" axis are 433MHz and 434MHz, respectively. Therefore, there is no wave interferences between transmitter and receiver, and transmitted data to microcontroller ATMEGA2560, which has several serial ports for transmission and receiving, are sent separately. Figure 5 shows the architecture of system images wireless receiver part.

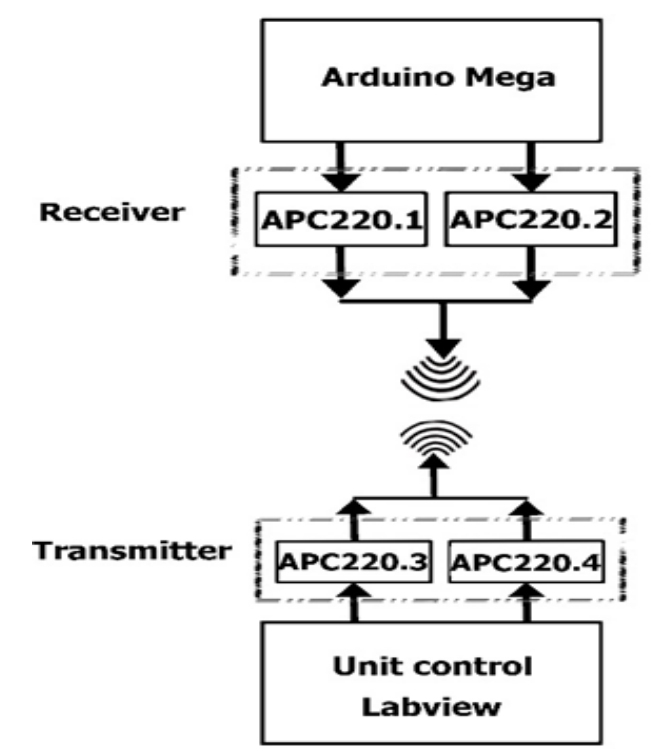

Fig. 5: Architecture of System Images Wireless Receiver Part.

Figure 6 shows the flowchart of overall operation and hardware. 


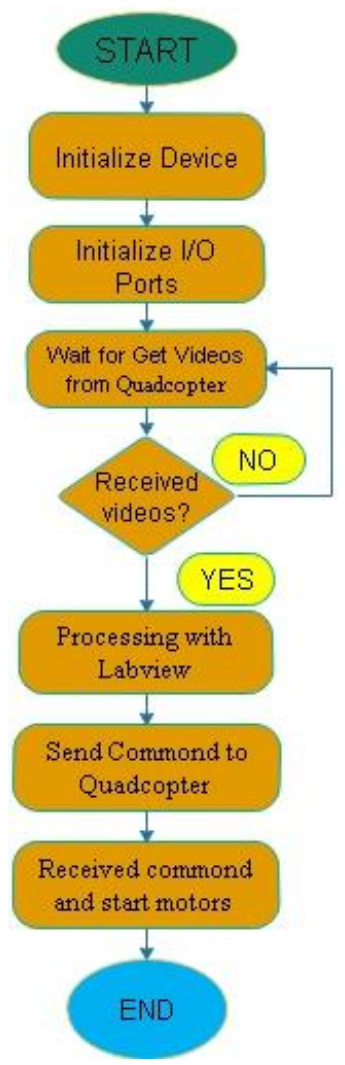

Fig. 6: System Flowchart.

For calibration we used the camera with a resolution of $492 \times 510$ pixels and the following standard model:

$p=R(P+t)$

Where, "p" is the point on the image plane, " $\mathrm{R}$ " is the rotation matrix, "P" is the point in the world frame and "t" is the translation vector and the translation vector is:

\section{$t=\left[x_{\text {CAM }} y_{\text {CAM }} 0\right]$}

Figure 7 shows how to adjust camera calibration including axis, target and image central point calibrations[5].

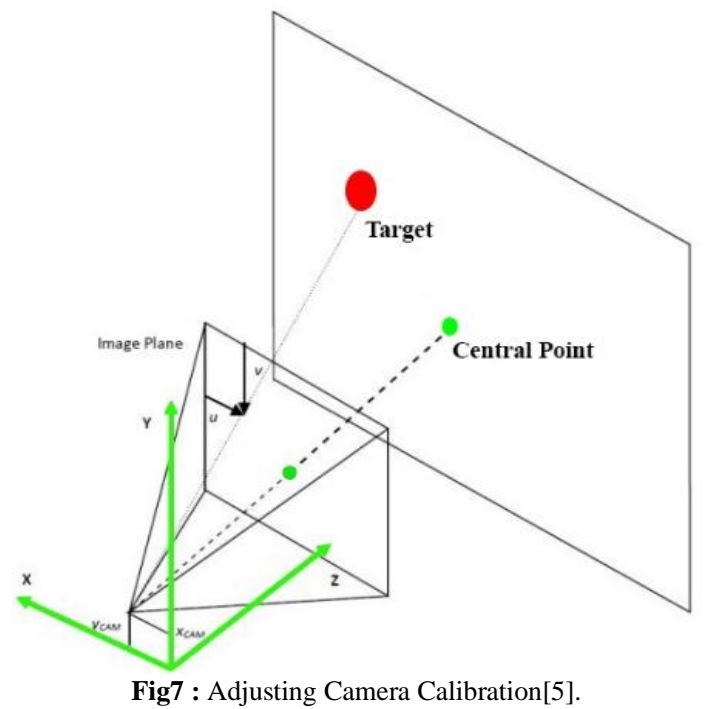

Figure 8 show the range of designed controller, which is the main processor embedded on the quadcopter. One of the most advantages of modular range and being metalized is that all parts can be separated or can be replaced by more advanced ones. 


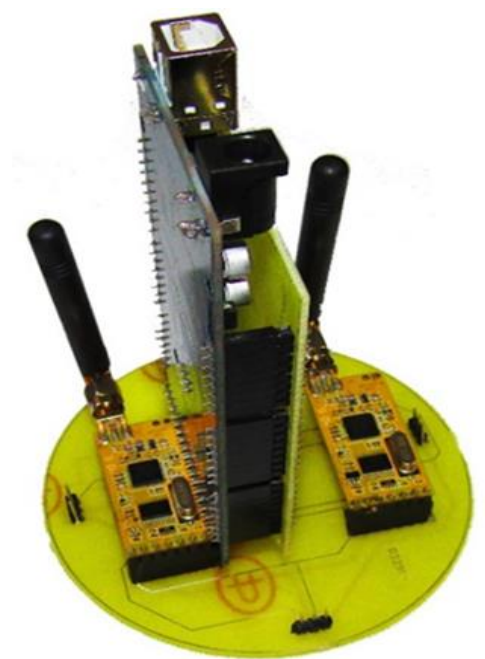

Fig. 8: Main Controller Range Embedded on the Quadcopter (Control Section).

As it can be seen from board image, after receiving Labview data by APC220 receiver-transmitter, brushless motors connected to board, rotated around " $\mathrm{X}$ " and "Y" directions. For moving quadcopter in right and left directions (X axis) two brushless motors are embedded and for moving quadcopter in up and down directions (Y axis), two brushless motors are also embedded. Figure 9 shows the control section.

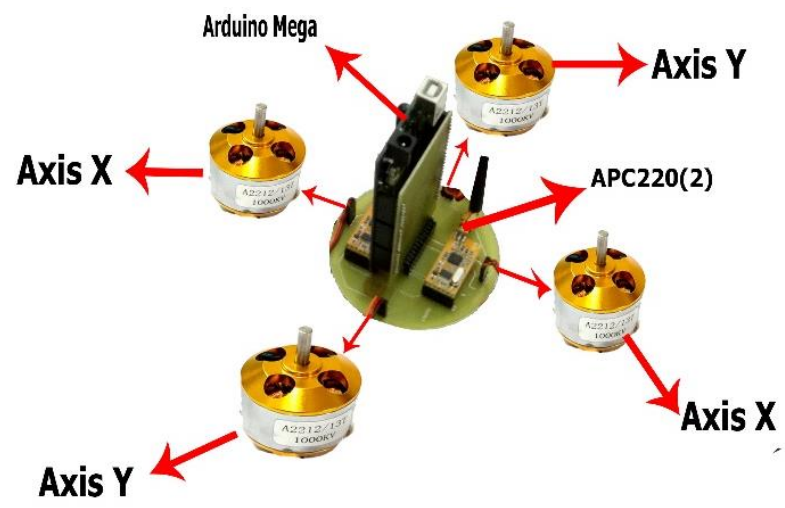

Fig. 9: Connecting Brushless Motors to Control Section.

\section{Results and discussion}

Mean shift is a technique for finding maximum points in a probability distribution function. This method can be used when there are discretized samples of desired function. This technique has iterative structure and converged to a maximum point by start with an initial guess. The aim of is to find mode and the initial guess is the location " $\mathrm{X}$ ". Now, points set which are in the neighboring of the current guess are considered, i.e. A little higher and a little smaller than " $X$ ". This neighboring points set is called "N(X)". Now, it is assumed that there is a function for better estimation of maximum location, which shows the importance of neighboring points, it means that, it allocates a weight to neighboring points. In statistics, this function is called kernel. It is clear that a kernel function can has different shapes. In following, a Gaussian kernel function is used, where its neighborhood weight of xi is defined as follows:

$\mathrm{k}\left(X_{i}-X\right)=e^{-c] \mid X_{1-x}} \|^{2}$

Now, a new guess of mode can be defined as follows,

$m(x)=\frac{\sum_{x_{1} E N(x)} R\left(x_{1}-x\right) x_{1}}{\sum_{x_{1} E N(x)} K\left(x_{1}-x\right)}$

Where, $\mathrm{m}(\mathrm{x})$ is the new guess. Now $\mathrm{m}(\mathrm{x})$ can be replaced by $(\mathrm{x})$ and repeat the algorithm. The finish condition is that the difference of $\mathrm{m}(\mathrm{x})$ in two consecutive iterates be negligible [6-8].

As mentioned earlier, in the present study LABVEIW and Mean shift are used for video process (Figure 10). Software has the capability that image processing and transmitting the required commands from serial port simultaneously. In the figure below, a graphical interface is shown for controlling and processing the target which tracking online the aircraft. After observing and selecting the target, a red box is draw around that by software and then information of " $\mathrm{X}$ " and "Y" axis which are both as code are transmitted to serial port. Brushless motors are moved by these two codes and therefore quadcopter is guidance toward target. 


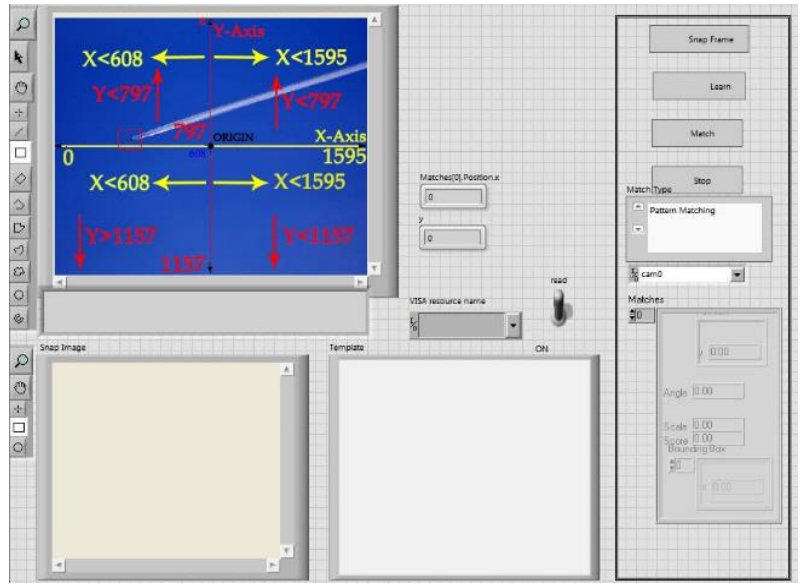

Fig. 10: User Interface for Video Processing.

As can be seen from Figure 10, received image from quadcopter is divided in to two main axis " $\mathrm{X}$ " and " $\mathrm{Y}$ " in the main frame, where the range of "X" axis is from 0 to 1595 and the range of "Y" axis is from 0 to 1157 . Table 1 shows the values related to each part of image.

Table 1: Divided Parameters of Screen

\begin{tabular}{lcc}
\hline Axis & Minimum value (left and up sides) & Minimum value (right and down sides) \\
\hline $\mathrm{X}$ & 0 & 1195 \\
$\mathrm{Y}$ & 0 & 1157 \\
\hline
\end{tabular}

For each of these "X" and "Y" axes, two brushless motor are defined. Transmitted values of "X" and "Y" axes to serial ports, are used for guidance quadcopter to left-right and up-down, respectively. To clarify, the target motion in the sight of quadcopter is investigated using graph. Figure 11 shows the different positions of target in the marked points; air targets in these points are shown and investigated.

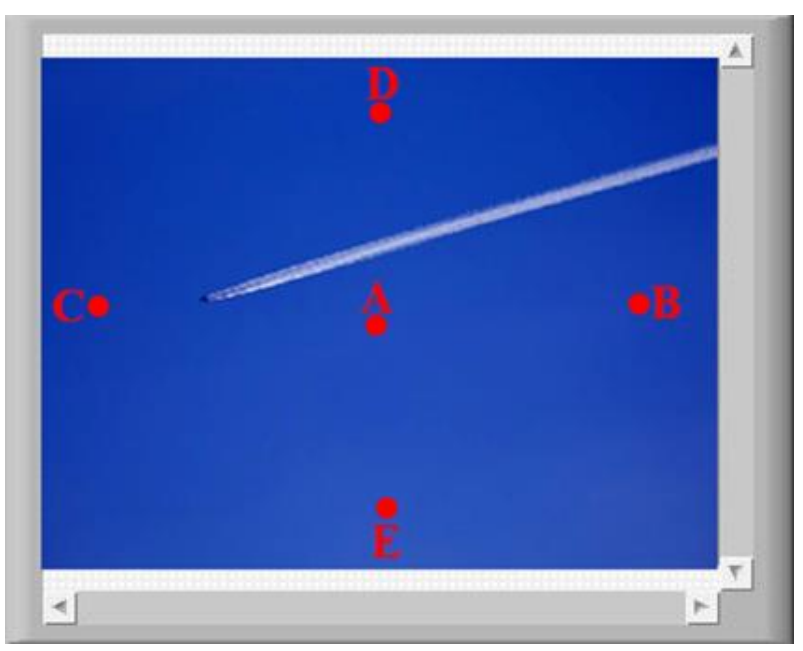

Fig. 11: Target Investigation in the Marked Points.

Figure 12 is related to software output, in a case which target is located in the center of screen, point "A". The aim of present study is that with every target motion in the screen, brushless motors also moved the quadcopter and put the quadcopter in direction with target, therefore the target position is returned to "A" point in the screen.
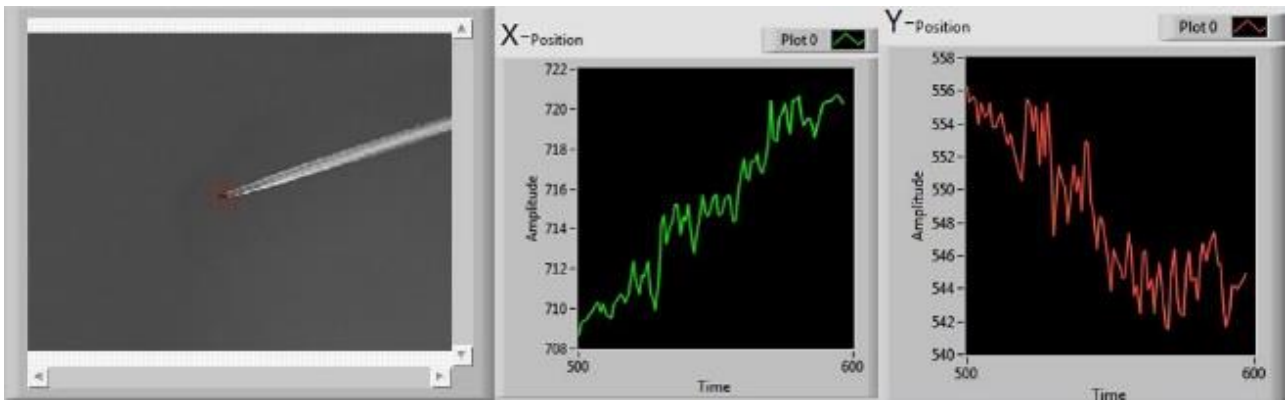

Fig. 12: Target Position at "A" Point.

"B", "C", "D" and "E" points are investigated in Figure 13, Figure 14, and Figure 15 and Figure 16. As it can be seen on each figure, target position has " $\mathrm{X}$ " and "Y" axes. 

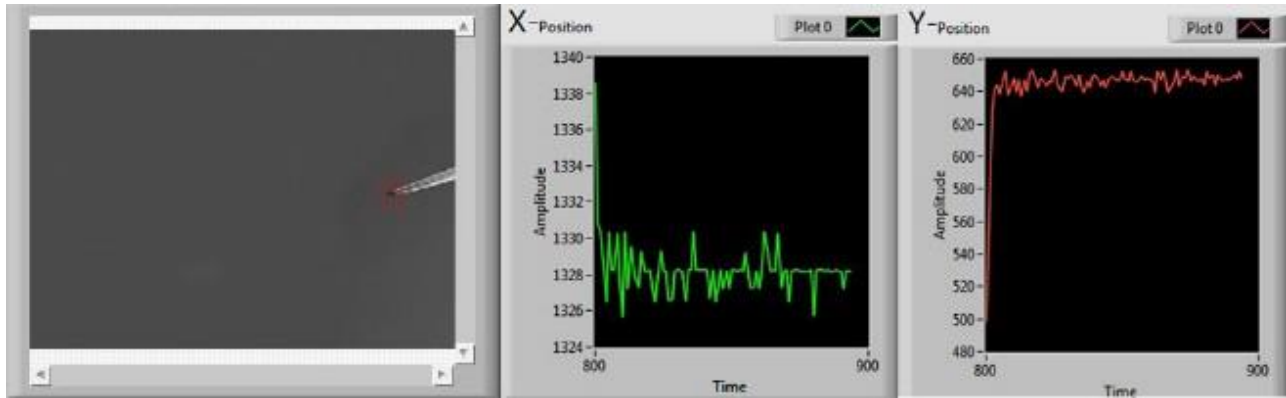

Fig. 13: Target Position at "B" Point.

During image process, blue background of sky becomes gray and target becomes dark. This led to easier detection of target. In the present study, shapes of target and background have not physical changes. At the time of presence of target at point "B", "X" axis has value between 1328 and 1330, which is sent to microcontroller via USB port and this axis is changed and shifted with target displacement. "Y" axis has also a value between 640 and 660. In order to run the brushless motors, knowing this range of values is no enough and having a constant value is no necessary; it means that motion of brushless motors is easily done by defining the values range from 640 to 660 . "C" and " $\mathrm{B}$ " points are related to " $\mathrm{X}$ " axis and the position of quadcopter is changed to right and left using this axis.
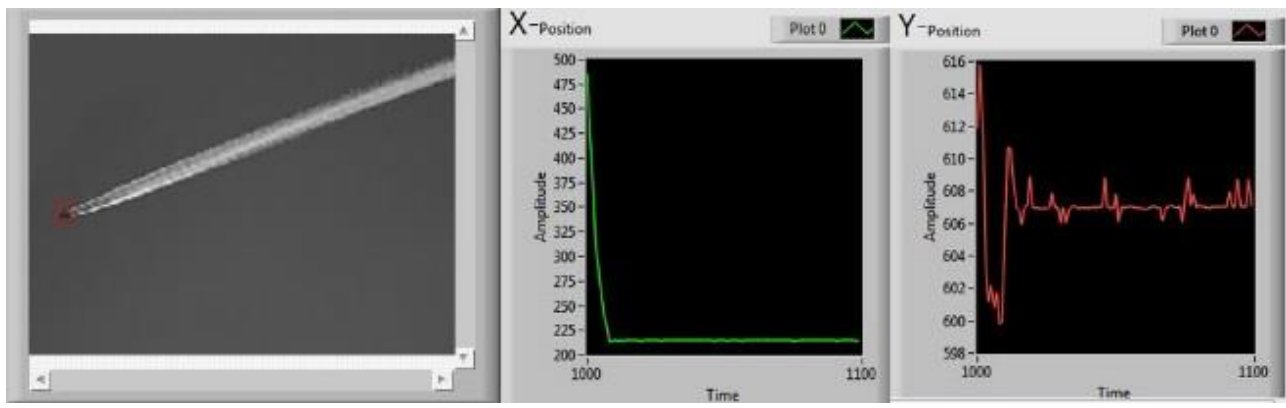

Fig. 14: Target Position at "C" Point.

Figure 15 shows the target position at point "D". This figure is related to quadcopter variations in "Y" axis. At the moment of positioning of target at point "D", both "X" and "Y" outputs are available. As can be seen from the figure, the range of "Y" output is between 200 to 250 and by and as it gets higher it gets closer to 0 . To control the quadcopter and gets higher in "Y" direction, the parameters of this axis can be sent to microcontroller, so that the target again get back to point "A".
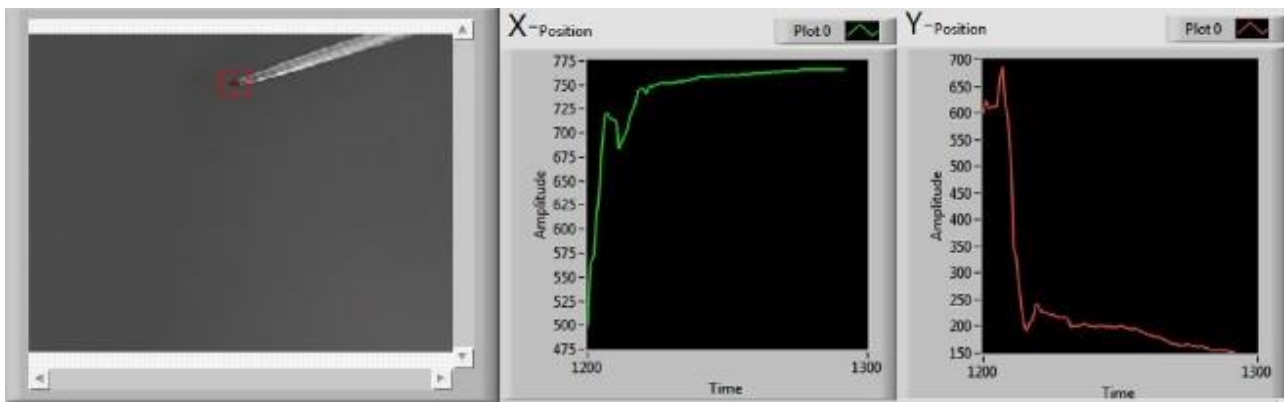

Fig. 15: Target Position at "D" Point.

Also, by positioning the target at point E, the "Y" output is the value between 985 and 986 (Figure 16). When the target positioned at this point, brushless motors dedicated for "Y" axis on the quadcopter nose turned on and the quadcopter move upward and target again get back to point "A". if the target simultaneously changed in both " $X$ " and "Y" axes, all brushless motors on the "X" and " $Y$ " axes are turned on separately and the quadcopter moves in both right-left direction ( $\mathrm{X}$ axis) and up-down direction (Y axis).
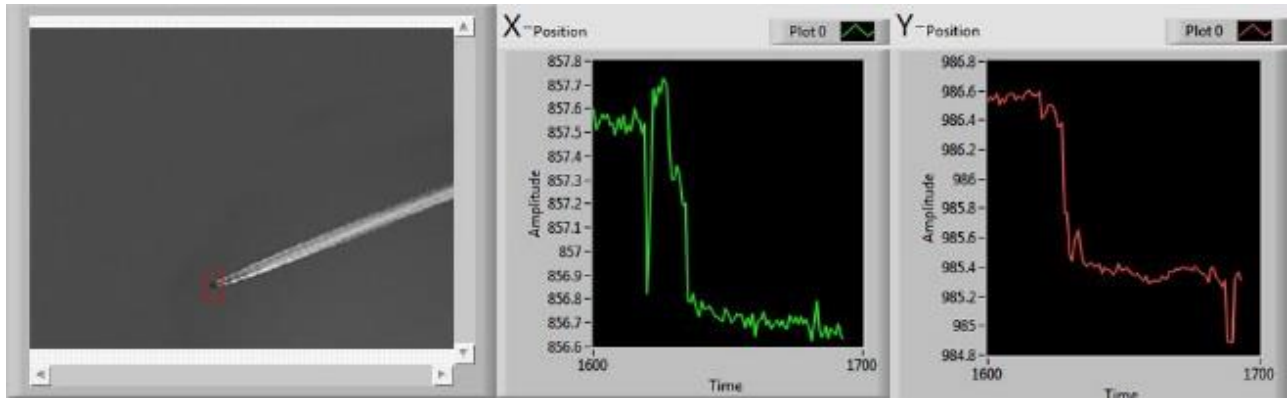

Fig. 16: Target Position at "E" Point.

Figure 17 shows the results of present study during target motion from point "A" to point "H". Recorded data and changed values are sent to microcontroller and brushless motors of " $\mathrm{X}$ " and "Y" axes are turned based on the received values. Until the target is not positioned at point "A". Until the target do not positioned at point "A", those process for each part of screen and for each selected target is repeated. 


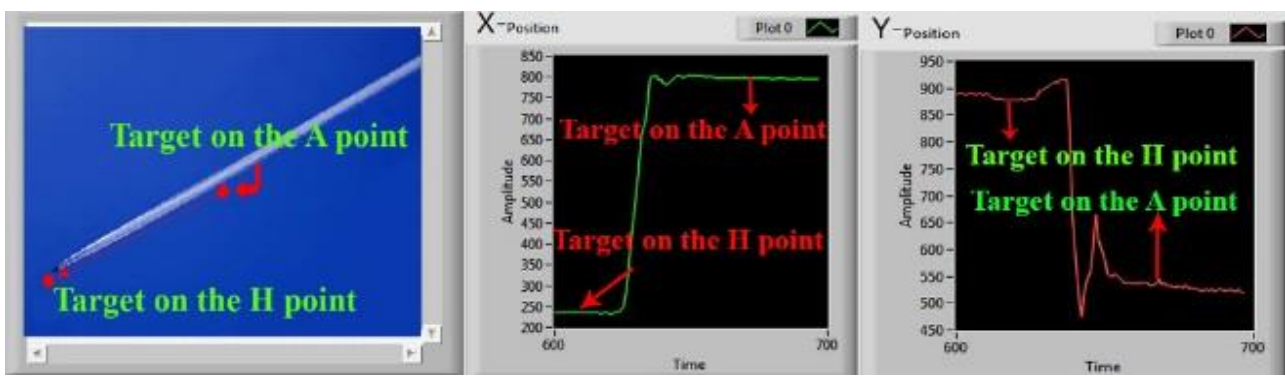

Fig. 17: Path Change of Target from Point "A to Point "H".

As can be seen from Figure 18, after positioning target at point "A", the values of both "X and "Y" axes are changed from 240 to 800 and from 890 to 550, respectively. This process is repeated for all image points, which means that quadcopter changes its direction until positioning target at point " $A$ ", so that quadcopter be in line with target. These values are sent to microcontroller embedded on the quadcopter by wireless. Also, microcontroller based on the predefined program, turns on the brushless motors and. In the present study, selection and tracking operations of a target were done in sunny day with temperature $30^{\circ} \mathrm{C}$.

In order to detect and define the type of air targets, the measuring body distance tab NI VISA of Labview software was used. In the present study, the targets which were defined earlier for software, were measured in different distances and were defined as a library for quadcopter, in order to detect military and civilian targets at different distances. To be able to detect the distance between target and quadcopter, longrange distance lasers was used. Figure 18 show a measured air target at distance $3 \mathrm{Km}$.

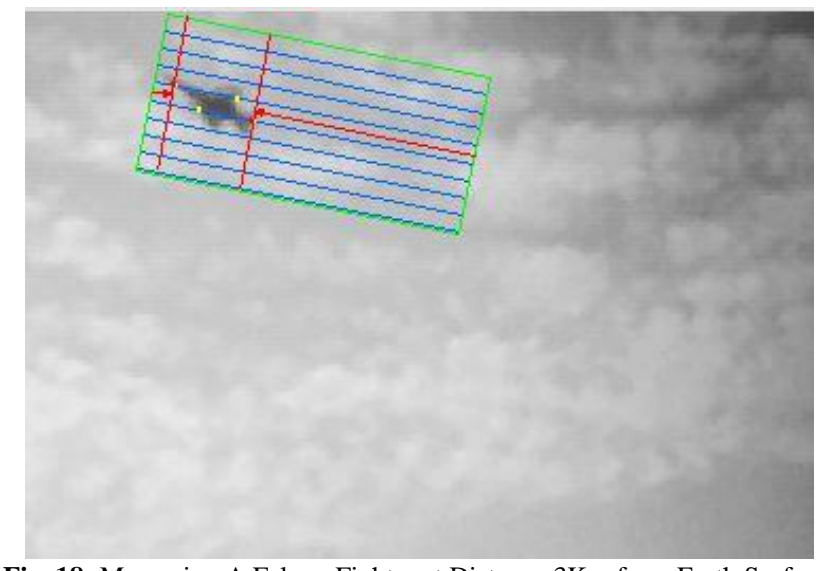

Fig. 18: Measuring A Falcon Fighter at Distance 3Km from Earth Surface.

This fighter is at distance $3 \mathrm{Km}$ and has the length $52.7 \mathrm{~mm}$. This size is different in different distances, and changes by getting closer and further of quadcopter camera sight. In Figure 19 a fighter and an airplane in a same distance with quadcopter are detected and civilian and military type is investigated.

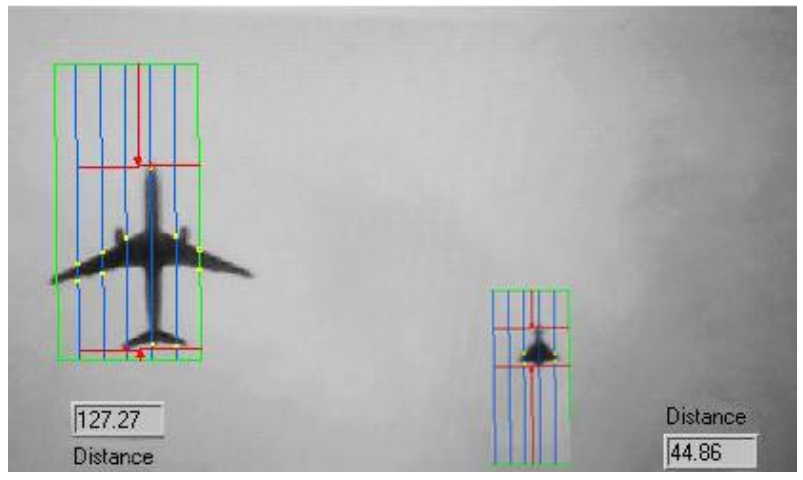

Fig. 19: Detection of Military and Civilian Aircraft.

As can be seen from Figure 20, using the object distance detection property in Labview software, the size of both aircrafts are investigated, where in this case, the length of military and civilian sample were $44.86 \mathrm{~mm}$ and $127.27 \mathrm{~mm}$. In this case, the camera sight is investigated in all distances and the quadcopter has the ability to differentiate between military and civilian aircrafts based on their length and engaged with military aircraft using this information. Also, aircraft dimensions in terms of their airfoil length can be investigated.

\section{Conclusion}

In the present study, a new system was designed and implemented using image process with Labview software, in order to tracking the air target and guidance quadcopter. Target detection and selection process was done by image process and by brushless motors quadcopter directed tracked the target. As an example, a target was detected and tracked in a sunny day with temperature $30^{\circ} \mathrm{C}$. The aim of study was that, in every condition, the target remained in the center of screen, so that the quadcopter and target be in line. In addition, during the quadcopter movement, quadcopter changed its path using " $\mathrm{X}$ " and " $Y$ " axes brushless motors, so that the target remained at point A. also, 
for detecting the military and civilian targets, distance detection property was used, to measure the length dimensions of fighters and passenger airplanes and comparing them with predefined values, it engaged with military aircraft.

\section{Acknowledgement}

The authors would like to thank the Shahid Sattari Aeronautical University of Science and Technology for the support of this research project.

\section{References}

[1] Naveenkumar. R, Pandiyan K, Prakash M, Saravanaperumal V, Jeya anusuya S,” Missile Burst Prevention and Tracking System Using Image Processing In MATLAB”, IJIRSET, Vol. 8, No. 2, (2019), http://www.ijirset.com/upload/2019/spl2/8.pdf.

[2] Vimal. M, Kumar. R, "Tracking of Ballastic Missiles and Velocity Measurement using Frame Distance Manipulation", Conference on Advances in Communication and Control Systems, (2013), https://pdfs.semanticscholar.org/98fe/fae92e61bafc91297ed47ffdc794860f4801.pdf.

[3] Zhongyuan. C, Xiaoming. L, Wanchun. C," Design of Real-Time Hardware-in-the-Loop TV Guidance System Simulation Platform”, International Journal of Aerospace Engineering, Vol. 2017, No. 11, (2017), pp. 1-16, https://doi.org/10.1155/2017/7834395.

[4] Jing. H, Ru. X, Ming, J, Guang. Z," Application of Genetic Algorithm in Target Image Processing of TV Guided Missile”, International Conference on Electronic, Information and Computer Engineering, Vol. 44, (2016), pp. 4, https://doi.org/10.1051/matecconf/20164401021.

[5] Kit. A, Ben. I, Michael. W, "A self-targeting missile system using computer vision”, MTRX 4700 Experimental Robotics Major Project University of Sydney.

[6] Dorin. C, Peter. M, "Mean Shift: a Robust Approach toward Feature Space Analysis", IEEE Transactions on Pattern Analysis and Machine Intelli gence, Vol. 24, No. 5, (2002), https://doi.org/10.1109/34.1000236.

[7] K. Fukunaga, L. Hostetler," The Estimation of the Gradient of a Density Function, with Applications in Pattern Recognition", IEEE Transactions on Information Theory, Vol. 21, No. 1, (1975), https://doi.org/10.1109/TIT.1975.1055330.

[8] Xiangru. L, Zhanyi. H, Fuchao. W," A note on the Convergence of the Mean Shift", Pattern Recognition, Vol. 40, No. 6, (2007), https://doi.org/10.1016/j.patcog.2006.10.016. 